\title{
A 3D MODEL OF QUASI-STATIC BRAIN MECHANICS: APPLICATION TO TUMOR-BEARING IMAGE REGISTRATION.
}

\author{
Stelios K. Kyriacou and Christos Davatzikos \\ Radiology, Johns Hopkins University, School of Medicine, Baltimore, Maryland \\ kyriacou@cbmv.jhu.edu
}

\begin{abstract}
A 3D biomechanical model of the human brain is being developed. Currently, the model is used to predict tumor growth "mass effect" (movement of brain tissue due to the tumor) and, thus, to improve image registration of various normal to tumor-bearing images.

INTRODUCTION Brain biomechanics has been investigated by many researchers for more than 30 years now. In particular, finite element analysis and other powerful computational methods have long been used in the investigation of head trauma to provide quantitative re sults. Nevertheless, the potential of these methods to simulate and predict the outcome of neurosurgical procedures and neuropathological processes has only recently been explored. The human brain is a soft yielding structure enclosed in a stiff casing, the skull. It has four fluid-filled cavities (ventricles) that communicate with each other as well as with the surface of the brain (subarachnoid space). The basic structures of the brain are the cerebral hemispheres and the cerebellum, separated by the dura (which adheres to the skull), falx and tentorium, stiff membranes that constrain movement of the brain tissue. In previous work, we have developed a two-dimensional model of brain tumor growth and used it for the preliminary registration of an anatomical atlas to the tumor-bearing (and thus locally deformed) patient image for use in radiosurgical planning [1]. In this manuscript, we present the extension of the above work to the $3 D$ domain.
\end{abstract}

METHODS The development of a 3D mesh together with the modeling of the boundary conditions is a very time consuming process. As a first approach, we have been using a digital (with stair-like boundary) mesh generation program developed by Dr. F. Kruggel at the Max-Planck-Institute of Cognitive Neuroscience, Leipzig, Germany. We use a hyperelastic compressible Mooney Rivlin material. Values for the material parameters are taken from the literature. Boundary conditions are imposed with the outer surface of the brain fixed and the ventricles assumed to have zero pressure.

RESULTS Preliminary results are shown in the figure. This is a section through the tumor region to show the displacements due to the tumor expansion. The image on the left is the undeformed configuration. Contact ele- ments (lighter gray areas) have been applied next to the ventricle areas (dark outlines) to avoid the possibility of inter-penetration of the ventricle sides. The image on the right is the deformed configuration. The tumor area is represented by the dark dots area on the left side of the images. The mesh shown is coarse, mostly for demonstration purposes.

We are developing a more detailed/accurate $3 \mathrm{D}$ brain mesh/model through the use of a digitized anatomical atlas from which we can easily extract isosurfaces (a capability provided by the new matlab 5.3). We will follow with appropriate boolean geometry operations to create the right solid and finally mesh it using commercial CAD software.

DISCUSSION The material model was chosen to be simple enough for our specific application of tumorbearing image registration. Many refinements are in order to make the model more general for other quasistatic application. In particular, the material model will be upgraded to a poroelastic type and will include metabolic water production in the tissue, capillary effects, cerebrospinal fluid creation and absorption, and fluid movement through the various tissues.

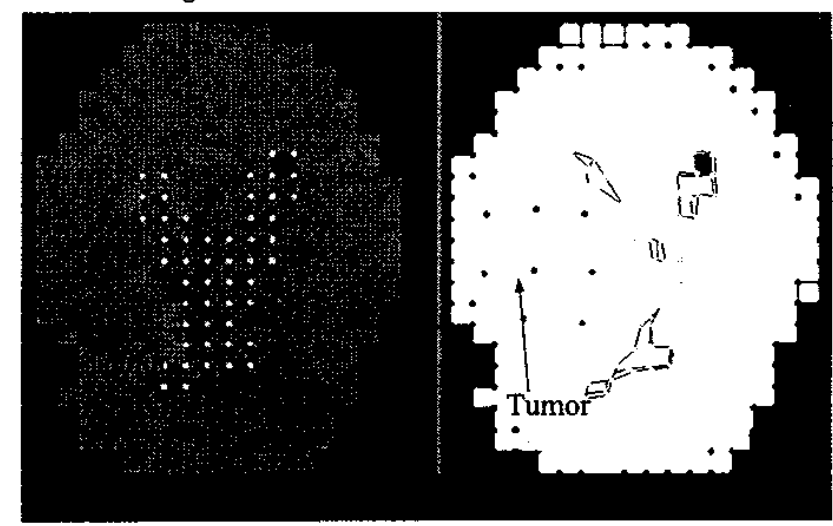

\section{References}

[1] S.K. Kyriacou, C. Davatzikos, S.J. Zinreich, and R.N. Bryan. Nonlinear elastic registration of brain images with tumor pathology using a biomechanical model. IEEE Trans. on Med. Imag. Submitted. 